\title{
Determinants of impaired bed mobility in Parkinson's disease: Impact of hip muscle strength and motor symptoms
}

\author{
Seira Taniguchi ${ }^{\mathrm{a}, \mathrm{b}, *}$, Nicholas D'cruz $^{\mathrm{c}}$, Miho Nakagoshi ${ }^{\mathrm{d}}$, Toshinori Osaki ${ }^{\mathrm{d}}$ and Alice Nieuwboer \\ ${ }^{a}$ Center for Information and Neural Networks (CiNet), Advanced ICT Research Institute, National Institute of \\ Information and Communications Technology (NICT), Suita, Japan \\ ${ }^{\mathrm{b}}$ Osaka University Graduate School of Medicine, Suita, Japan \\ ${ }^{\mathrm{c}}$ Department of Rehabilitation Sciences, Neurorehabilitation Research Group, KU Leuven, Leuven, Belgium \\ ${ }^{\mathrm{d}}$ Department of Rehabilitation, Mikiyama Rehabilitation Hospital, Miki, Japan
}

Received 3 November 2021

Accepted 11 January 2022

\begin{abstract}
.
BACKGROUND: Although most patients with Parkinson's disease (PD) present difficulties of bed mobility, the contributing factors to impaired bed mobility in PD are unknown.

OBJECTIVE: To compare bed mobility and muscle strength between PD patients and healthy controls, and investigate the determinants of bed mobility in PD.

METHODS: Sixteen patients with PD and ten age- and sex-matched healthy controls (HC) were enrolled. Time and pattern to get out of bed to their preferred side at usual speed, muscle torque in lower extremities and motor symptom burden were also measured.

RESULTS: PD exhibited significantly slower speed in bed mobility and lower torque in the hip adductor/abductor/flexor muscle than HC. Slower movement time in PD was correlated with weaker hip adductor torque on the more affected side $(\mathrm{Rs}=-0.56, p<0.05)$ and with higher score in arm rigidity both sides ( $\mathrm{Rs} \geq 0.79, p<0.01)$. There were no significant differences between the categorised movement patterns and movement time in $\operatorname{PD}(p=0.31)$.

CONCLUSIONS: Reduced hip adductors torque and severe arm rigidity are associated with slowness of getting out of bed, implying that these components could be used as targets for rehabilitation practice to improve bed mobility in PD.
\end{abstract}

Keywords: Parkinson's disease, bed mobility, hip muscle strength, rigidity, rehabilitation, quality of life

\section{Introduction}

Parkinson's disease (PD) is a progressive neurodegenerative disorder and is clinically characterised by bradykinesia, rigidity, tremor, and postural instability (Gelb et al., 1999; Jankovic, 2008). Difficulties in bed mobility are common problems in patients with PD (Aragon \& Kings, 2018; Nieuwboer et al., 1998; Wimmers \& Kamsma, 1998), and resolving these

*Address for correspondence: Seira Taniguchi, 1-4 Yamadaoka, Suita, Osaka 565-0871, Japan. E-mail: seira.taniguchi. pt@gmail.com. limitations are a prerequisite for functional independence. Notably, a recent longitudinal study showed that difficulties in turning in bed begin 7 to 11 years before PD diagnosis (Fereshtehnejad et al., 2019). Once PD has been diagnosed it is often one of the first difficulties noted (Pfeiffer \& Wszolek, 2012) and worsens with advanced disease (Mirelman et al., 2020).

Moreover, urinary frequency and urgency are also common in patients with PD due to poor control of the detrusor muscle, arising from disease-related deficits of the autonomic nervous system (Yeo et al., 2012). Collectively, the worsening of bed mobility and uri- 
nary symptoms significantly impact patient comfort at night, especially because the effect of medication is then at its lowest (Viwattanakulvanid et al., 2014). Therefore, increasing insight into the underlying difficulties of bed movements in PD is crucial to improve patients' quality of life.

Earlier studies have already reported on the movement patterns (Mount et al., 2009) and turning strategies (Stack \& Ashburn, 2006) used by PD patients to get out of bed. In addition, sensor-based analysis noted a slowed speed in turning in bed (Bhidayasiri et al., 2016; Bhidayasiri et al., 2017; Chiang et al., 2018; Mirelman et al., 2020; Sringean et al., 2016). Furthermore, other studies have shown that muscular weakness slowed the performance of single-joint movements in patients with PD (Robichaud et al., 2004; Wierzbicka et al., 1991). Nevertheless, contributing factors such as muscle strength and limb rigidity to difficulties in getting out of bed have not been previously considered.

In line with these previous reports, it is conceivable that muscular weakness or stiffness may influence movement time as well as movement patterns and thereby impact the ease with which patients with PD can get out of bed.

Therefore, the aims of this study were 1) to evaluate movement time and the movement patterns of getting out of bed and the muscle strength in patients with PD, and compare the results with age- and sex-matched healthy controls and 2) to investigate whether muscle strength as well as motor symptoms on the affected and unaffected body sides could influence movement time to get out of bed.

We hypothesised that PD patients would exhibit a slower bed mobility and weaker lower muscle strength than that of healthy controls, and a reduced muscle strength and severe motor symptoms especially on the more affected side would be related to slowed bed mobility in patients with PD. The results of this study will provide useful insight into the nature of difficulties with bed mobility in patients with PD.

\section{Methods}

\subsection{Study design and ethics}

This study was approved by the Mikiyama Rehabilitation Hospital ethics committee (approval number: 201502) and was conducted in accordance with the Declaration of Helsinki. We obtained written informed consent from each participant after explain- ing the purpose, expected benefits, and potential harm of this research.

\subsection{Participants}

Sixteen patients with PD and ten age- and sexmatched healthy controls (hereafter referred to as HC) were enrolled in this study. The inclusion criteria for PD were as follows: (1) a diagnosis of idiopathic PD in accordance with the MDS clinical diagnostic criteria (Postuma et al., 2015), (2) Hoehn and Yahr stage 2-4; (3) signs of difficulties in bed mobility as defined by a score of $>0$ for item 11 ("getting out of bed without a cover") in the Modified Parkinson Activity Scale (M-PAS) (Keus et al., 2009; Taniguchi et al., 2021), (4) a score of the Mini Mental State Examination (MMSE) $\geq 24$ (Folstein et al., 1975). The exclusion criteria for PD were (1) a history of other neurologic or musculoskeletal disorders and severe orthopaedic disease; (2) any pain that affected the movement patterns for getting out of bed; and (3) the presence of dyskinesia as assessed using the Movement Disorder Society-sponsored revision of the Unified Parkinson's Disease Rating Scale (MDSUPDRS) Part III (Goetz et al., 2007). All patients with $\mathrm{PD}$ were assessed in self-reported ON medication state.

\subsection{Getting out of bed task}

The subjects were asked to get out of bed to their preferred side at a usual speed without a bed cover. They were instructed to get up from lying in the supine position and come to sit at the side of the bed with their legs on the floor, following the same protocol as in the M-PAS (Keus et al., 2009). All performances were video recorded and timed. The two investigators (ST and TO) categorised the total movement patterns of getting out of bed based on previously reported criteria (Mount et al., 2006; Stack \& Ashburn, 2006), with disagreements resolved through discussion. Supplementary Figure 1 (A, B, and C) shows that the categorisation captured the movement patterns of leg movement and trunk rotation as follows: "Trunk lateral flexion with rotation", "Trunk lateral flexion without rotation", and "Sit and hip-hitching".

\subsection{Test of muscle strength}

The muscle strength of the hip flexors, extensors, abductors and adductors, knee flexors and extensors, ankle plantar flexion, and dorsiflexion was mea- 
sured during isometric muscle contraction using a hand-held dynamometer (HHD; $\mu$-Tas F1, ANIMA Co. Ltd), applying the HHD with a belt-fixation method (Thorborg et al., 2013). The torque (Nm) was obtained by multiplying the force $(\mathrm{N})$ by the lever arm $(\mathrm{m})$ of each participant as a unit of the muscle strength. We also evaluated the intra-rater reliability of the values for muscle strength.

\subsection{Motor symptom measurement}

The motor symptoms from MDS-UPDRS Part III were assessed in a standardised manner and item-scores were summed into four motor symptom components in accordance with previous studies (Bouça-Machado et al., 2020; Lau et al., 2019): akinesia: sum of sub-items 3.2, 3.4-3.8, 3.14; rigidity: sum of sub-item 3.3; tremor: sum of sub-items 3.15-3.18; and axial signs: sum of sub-items 3.1, 3.9-3.13. Furthermore, the more affected side was defined by initial motor symptoms onset in PD, while the less affected side was defined by the other side of the more affected side. The MDS-UPDRS Part III was administered by the physical therapist (ST) who has completed the MDS-UPDRS online training.

\subsection{Statistical analysis}

The tests for muscle strength and getting out of bed were performed twice and two values were averaged.

For intra-rater reliability, we calculated the intraclass correlation coefficient (ICC) (Koo \& Li, 2016) of muscle strength, and Cohen's kappa coefficients (McHugh, 2012) of the movement patterns of getting out of bed. For comparing between-group differences, the Mann-Whitney U test was used in age, movement time, muscle strength, and MMSE scores, while Fisher's exact test was used in sex and movement patterns.

Spearman's rank correlation coefficient (Rs) was used to assess relationship between movement time and other measures, and correlation ratio $(\eta)$ was calculated for correlation between movement time and movement patterns. After checking for multi-collinearity (variance inflation factor $>2.5$ ), a multivariable backward linear regression model was built to investigate the determinants of movement time. Dwass-Steel-Critchlow-Fligner test for multiple comparisons analysis were used to assess the differences among the three movement pattern groups. All statistical analyses were performed using software R (R Foundation for Statistical Computing;
$\mathrm{R}$ package version 4.0.2). A $p$-value of $<0.05$ was considered statistically significant.

\section{Results}

\subsection{Demographic information}

The patient characteristics are shown in Table 1. There were no significant between-group differences in age and sex $(p>0.05)$.

\subsection{Group differences in lower muscle strength}

Supplementary Table 1 summarises the muscle strength in lower extremities and intra-rater reliability. PD patients had significantly weaker hip, knee and ankle flexors as well as hip abductors and adductors $(p<0.05)$. The results showed excellent agreement in both PD (ICC $=0.95-0.99)$ and $\mathrm{HC}$ (ICC $=0.96-0.99$ ), suggested that the obtained scores were consistent. Additionally, the movement patterns of getting out of bed (Cohen's kappa coefficient $=1$ ) indicated almost perfect agreement.

\subsection{Movement time and patterns for getting out of bed}

Figure 1 shows between-group differences in movement time. Compared with HC, PD patients

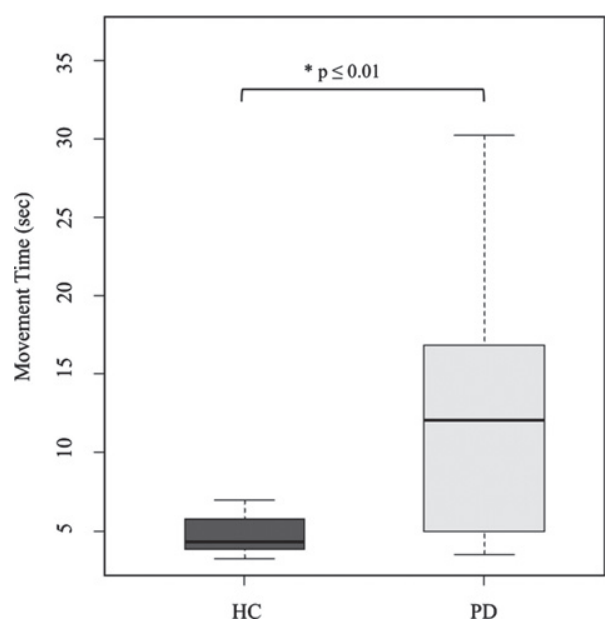

Fig. 1. Between-group comparison of movement time of bed mobility.

Asterisks indicate significant pair-wise differences (MannWhitney $\mathrm{U}$ test, $\left.{ }^{*} p \leq 0.01\right)$. HC $=$ age- and sex-matched healthy controls; $\mathrm{PD}=$ patients with Parkinson's disease. The boxplot shows between-group difference in movement time of getting out of bed. The PD group showed significantly slower speed in getting out of bed. 
showed significantly lower speed in getting out of bed $(p \leq 0.01)$. Further, there were significant betweengroup differences in movement patterns $(p<0.05)$.
Table 1 shows that a larger proportion of PD patients used the trunk lateral flexion without rotation pattern $(62.5 \%)$, while $\mathrm{HC}$ mostly used the trunk lateral

Table 1

Participant demographics and clinical details of patients with Parkinson's disease

\begin{tabular}{|c|c|c|c|}
\hline Variables & $\begin{array}{c}\text { PD } \\
(n=16)\end{array}$ & $\begin{array}{c}\mathrm{HC} \\
(n=10)\end{array}$ & $p$-value \\
\hline Age (years) & $73.4 \pm 8.8$ & $73.0 \pm 9.5$ & 0.66 \\
\hline Sex (males/females) & $6 / 10$ & $4 / 6$ & $1^{a}$ \\
\hline Disease duration (years) & $10.1 \pm 5.6$ & & \\
\hline Hoehn and Yahr stages & $\mathrm{II}=6 \% ; \mathrm{III}=50 \% ; \mathrm{IV}=44 \%$ & & \\
\hline MDS-UPDRS Part III (0-132) & $35.4 \pm 11.3$ & & \\
\hline $\operatorname{MMSE}(0-30)$ & $27.3 \pm 1.3$ & & \\
\hline Movement time (sec) & $12.9 \pm 8.4$ & $4.7 \pm 1.1$ & $\leq 0.01^{*}$ \\
\hline \multicolumn{4}{|l|}{ Movement pattern } \\
\hline - Trunk lateral flexion with rotation & $12.5 \%$ & $80.0 \%$ & $<0.05^{* a}$ \\
\hline - Trunk lateral flexion without rotation & $62.5 \%$ & $20.0 \%$ & \\
\hline - Sit and hip-hitching & $25.0 \%$ & $0 \%$ & \\
\hline
\end{tabular}

Significant results are shown in bold font. ${ }^{*} p<0.05$. Results of age, disease duration, movement time indicate mean \pm standard deviation; MMSE: Mini Mental State Examination; Movement time were averaged over two measurements, ${ }^{a}$ Fisher's exact test (Bonferroni corrected $p$-value).

Table 2

Relationship between the movement time of getting out of bed and the other scores in patients with Parkinson's disease

\begin{tabular}{llcc}
\hline & & \multicolumn{2}{c}{ Movement time (sec) } \\
\cline { 2 - 4 } Variables & Affected side & Rs & $p$-value \\
\hline Muscle strength & & & \\
Hip flexor torque & more & $-0.46^{a}$ & 0.06 \\
& less & -0.12 & 0.66 \\
Hip extensor torque & more & -0.09 & 0.75 \\
& less & -0.27 & 0.31 \\
Hip abductor torque & more & -0.38 & 0.15 \\
& less & -0.38 & 0.15 \\
Hip adductor torque & more & $-\mathbf{0 . 5 6}$ & $<0.05$ \\
& less & -0.22 & 0.41 \\
Knee flexor torque & more & 0.10 & 0.73 \\
& less & 0.26 & 0.35 \\
Knee extensor torque & more & -0.08 & 0.79 \\
Ankle plantar-flexion torque & less & 0.15 & 0.59 \\
Ankle dorsi-flexion torque & more & -0.19 & 0.47 \\
MDS-UPDRS Part III & less & -0.15 & 0.58 \\
Axial Signs & more & -0.18 & 0.51 \\
Akinesia & less & -0.20 & 0.46 \\
Tremor & & & \\
Rigidity total & & 0.30 & 0.26 \\
Rigidity neck & & $\mathbf{0 . 5 3}$ & $<0.05$ \\
Rigidity upper extremity & & 0.19 & 0.47 \\
Rigidity lower extremity & & 0.39 & 0.14 \\
Movement pattern & more & 0.15 & 0.58 \\
\hline
\end{tabular}

Significant results are shown in bold font. ${ }^{*} p<0.05,{ }^{* *} p<0.01 .{ }^{a} \mathrm{~A}$ trend towards significant correlation;

${ }^{b}$ Correlation ratio $(\eta)$. Rs $=$ Spearman's rank correlation coefficient; more $=$ more affected side; less $=$ less affected side. The scores of tremor, axial signs, and akinesia are defined using sub-items of the MDS-UPDRS Part III: axial signs: sum of sub-items 3.1, 3.9-3.13; akinesia: sum of sub-items 3.2, 3.4-3.8, 3.14; tremor: sum of sub-items 3.15-3.18; and rigidity: sum of sub-items 3.3. 
flexion with rotation pattern (80\%). A smaller group adopted sit and hip-hitching that was only found in the PD group (25\%), suggested PD-specific movement pattern. Moreover, those commonly adopted movement patterns by the PD group indicated these features when attempting to turn, without involving head-trunk rotation but using hands in turning such as "grasping the edge of the bed by the hand" in trunk lateral flexion without rotation pattern, or "pushing off with hands" in sit and hip-hitching pattern as seen in Supplementary Figure 1 (B, and C, respectively).

There was no significant correlation between movement time and the three movement patterns in PD. Further, multiple comparison analysis revealed that there were no significant differences between the three movement patterns and movement time in PD $(\mathrm{H}(2)=2.37, p=0.31)$, suggesting that there were no differences in movement time between participants using different movement patterns in PD patients.

\subsection{Relationship between bed mobility and other scores in $P D$}

Table 2 showed relationships between the movement time of getting out of bed and the other scores in PD patients, including the muscle strength measures on the affected and unaffected sides, scores in the MDS-UPDRS III total and in each of the four components (axial signs, akinesia, tremor, and rigidity), and movement patterns.

As for muscular strength, the movement time was inversely correlated with hip adductor torque on the more affected side $(\mathrm{Rs}=-0.56, p<0.05)$. It also tended to be related with reduced hip flexor torque on the more affected side ( $R s=-0.46, p=0.06$ ). Conversely, no relationship was observed between movement time and muscle strength in HC. These results indicated that weaker hip adductors and hip flexors slowed down performance and that this was PD-specific.

In addition, slower movement time was associated with higher scores in akinesia $(\mathrm{Rs}=0.53$, $p<0.05)$, and arm rigidity both on the less affected side ( $\mathrm{Rs}=0.79, p<0.01)$ and on the more affected side ( $\mathrm{Rs}=0.80, p<0.01$ ). Moreover, backward linear regression revealed that the rigidity score of the more affected arm remained the only independent predictor $(\beta=5.99, p<0.01)$ and explained $75 \%$ of the variability in the movement time.

Furthermore, multiple comparison analysis revealed no significant differences in akinesia $(\mathrm{H}(2)=$ $0.66, p=0.72)$, tremor $(\mathrm{H}(2)=0.54, p=0.76)$, axial sign scores $(H(2)=1.78, p=0.41)$, or rigidity total score $(\mathrm{H}(2)=5.56, p=0.06)$ suggesting that there were no differences in the severity motor symptoms among the three movement pattern groups in PD.

\section{Discussion}

To the best of our knowledge, this is the first detailed study to clarify contributing factors for the difficulties with getting out of bed in PD. The main findings of this study were that the more slowed movement times in PD compared to HC was highly associated with reduced hip adductor torque on the more affected side, and arm rigidity at both sides, as predicted by our initial hypothesis.

\subsection{Slowness in bed movement in $P D$}

PD exhibited significantly slower speed in bed mobility compared to $\mathrm{HC}$ which is consistent with previous study (Mount et al., 2009). In addition, a slower speed in PD was strongly related to akinesia, and not to axial signs. It is unsurprising that the severity of akinesia may directly reflect the slower performance in PD for getting out of bed. However, as trunk movement relies on axial mobility, it is surprising that no link with other axial symptoms were found. It could be that other axial symptoms, rely more on postural control and balance functions, which are less needed for movements carried out in a supine position.

\subsection{Reduced hip adductors torque is related to slowness bed movement in PD}

The slowed movement time was significantly related to reduction of hip adductors and also tended to be associated with reduction of hip flexors in PD. These key muscle groups were as much as $50 \%$ weaker in PD compared to HC, and may contribute to the slowness bed mobility in PD.

Regardless of the movement patterns used, hip adduction at the far side of the movement direction creates momentum for bringing the legs out of the bed. Moreover, it was reported that PD patients were more apt to lift both legs synchronously from bed compared to healthy elderly group (Mount et al., 2009) which might explain the greater hip flexors contribution in bed mobility in PD.

Furthermore, from a biomechanical perspective, the lower extremities account for the largest weight 
proportion (approximately $32 \%$ in both lower limbs) of the human body (Russell Merritt et al., 2005). Thus, the legs as a weight can assist in lifting the trunk to an upright position with less involvement of trunk functions (Mount et al., 2006).

Collectively, it was possible that adduction and flexion of both thighs together acted to unite the legs, which could be a precondition for dropping the legs off the bed. If that is the case, the reduced hip adductors and flexors subsequently could have slowed getting out of bed in PD; however, further kinematic or electromyographic analyses should be performed to clarify these points.

\subsection{Upper limb contribution for trunk rotation in $P D$}

In this study, arm rigidity on the more affected side statistically explained $75 \%$ of the variance in the movement time to get out of bed, suggesting it was one of the key contributors to slowness of bed mobility in PD. It is known that upper limb movement plays an important role in turning in bed in patients with a mild stage of PD having axial dysfunction (Lakke, 1985; Stack \& Ashburn, 2006). In the present study, most patients with PD adopted the movement patterns involving reduced trunk rotation and increased usage of hands while turning in bed.

These findings may explain the greater dependence on the use of upper limb movement in turning, as pertaining to people with mid-stage of PD as enrolled in our study. Thus, advanced arm rigidity and trunk inflexibility together could have played a role in the slowness of getting out of bed in PD.

\subsection{Selection of movement patterns in PD}

The most common strategy was trunk lateral flexion without rotation in PD, which is consistent with previous study (Mount et al., 2009). Additionally, sit and hip-hitching was a frequent PD-specific movement pattern in this study. The movement patterns required no trunk rotation, suggesting that these modes can be beneficial for patients with severe rigidity. In contrast, trunk lateral flexion with rotation was the least popular movement pattern in PD, possibly because it requires axial flexibility for rolling and for controlling the limbs sequentially. Therefore, this pattern may be possible only for patients with low rigidity or axial mobility problems.

\subsection{Therapeutic recommendations}

As for rehabilitation, adductor muscle strength may be a useful therapeutic target to improve bed mobility. Additionally, upper limb movement (e.g. shoulder horizontal adduction and the grasping the edge of the bed with the opposite hand) may be a helpful compensation for axial dysfunction to enable turning sideways and avoid having to rely on the hip flexors and abdominal muscles, which are weakened (Mount et al., 2006).

Involving limb movements and therapeutic strategies to reduce upper limb rigidity may facilitate turning and thus shorten the movement time to get out of bed. Furthermore, therapists should teach caregivers how to apply movement patterns which require a minimum of effort. As we described above, this could include recognition of using "unit" motion for the legs and pushing down by gravity, which facilitates trunk elevation can decrease caregiver burden, rather than using effort to lift up the upper body or rotate the trunk from supine to side-lying position.

\section{6. limitation}

This study had some limitations. First, the sample size was small and imbalance between the two comparison groups, and although we used a limited classification set of common movement patterns, the distribution of participants within these pattern-groups did not provide sufficient power for investigating differences. Larger samples with the same number of participants between two comparison groups should be included in future studies. Second, we did not perform behavioural tests in OFF medication state despite their low dopaminergic medication level at night. A follow-up study will be required to investigate bed mobility in OFF medication state especially in patients' real-life settings. Third, although we assessed only the strength of the lower limbs using a HHD with a belt-fixed method, an isokinetic dynamometer may be more suitable for assessing larger muscle groups. Further studies are required to include the trunk and upper limb muscles using an isokinetic dynamometer to investigate their contribution to bed mobility. However, despite these limitations, this study provides useful insights into the movement patterns and determinants of bed mobility in patients with PD. 


\section{Conclusion}

This study investigated determinants of bed mobility in PD, consisting of lower muscle strength on the more affected or less affected side and motor symptom severity. We found that slowed movement time was correlated with reduced hip adductor strength as well as with higher scores in arm rigidity on the more affected side. Our results suggested that reduction of hip adductors and severe arm rigidity are key contributing factors to slowed getting out of bed in patients with PD, which could be addressed by rehabilitation programs designed to improve bed mobility in PD. Further studies with larger sample sizes and assessing trunk and upper limb muscles are required to confirm the findings.

\section{Acknowledgments}

The authors thank all participants and partners of Mikiyama Rehabilitation Hospital. They are particularly grateful to Kyoko Machiba, Kimie Nakaichi, and Akiko Tsutsui for their assistance in recruiting healthy control participants. This work was partially supported by JSPS KAKENHI grant number JP21K17524.

\section{Conflict of interest}

None of the authors have any conflicts of interest to declare.

\section{Supplementary material}

The supplementary material is available in the electronic version of this article: https://dx.doi.org/ 10.3233/NRE-210301.

\section{References}

Aragon A, \& Kings J. Occupational therapy for people with Parkinson's, Second edition (2018). The Royal College of Occupational Therapists, London, UK, 54-56.

Bhidayasiri, R., Sringean, J., Taechalertpaisarn, P., \& Thanawattano, C. (2016). Capturing nighttime symptoms in Parkinson disease: Technical development and experimental verification of inertial sensors for nocturnal hypokinesia. Journal of Rehabilitation Research and Development, 53, 487-498.
Bhidayasiri, R., Sringean, J., \& Thanawattano, C. (2017). Impaired bed mobility: quantitative torque analysis with axial inertial sensors. Neurodegenerative Disease Management, 7, 235-243.

Bouça-Machado, R., Pona-Ferreira, F., Gonçalves, N., Leitão, M., Cacho, R., Castro-Caldas, A., Ferreira, J. J., \& and CNS Multidisciplinary Team. (2020). Outcome measures for evaluating the effect of a multidisciplinary intervention on axial symptoms of Parkinson's disease. Frontiers in Neurology, 11.

Chiang, S. L., Lin, C. H., Hsu, Y. D., Wei, S. H., Sung, W. H., Lu, L. H., Chang, S. T., Ho, T. Y., Shen, Y. P., Chen, L. C., \& Lin, C. H. (2018). Analysis of trunk rolling in Parkinson's disease patients using a mattress mobility detection system. Computer Methods and Programs in Biomedicine, 162, 157-163.

Fereshtehnejad, S. M., Yao, C., Pelletier, A., Montplaisir, J. Y., Gagnon, J. F., \& Postuma, R. B. (2019). Evolution of prodromal Parkinson's disease and dementia with Lewy bodies: a prospective study. Brain, 142, 2051-2067.

Folstein, M. F., Folstein, S. E., \& McHugh, P. R. (1975). "Minimental state". A practical method for grading the cognitive state of patients for the clinician. Journal of Psychiatric Research, 12, 189-198.

Gelb, D., Oliver, E., \& Gilman, S. (1999). Diagnostic criteria for Parkinson disease. Archives of Neurology, 56, 33-39.

Goetz, C. G., Fahn, S., Martinez-Martin, P., Poewe, W., Sampaio, C., Stebbins, G. T., Stern, M. B., Tilley, B. C., Dodel, R., Dubois, B., Holloway, R., Jankovic, J., Kulisevsky, J., Lang, A. E., Lees, A., Leurgans, S., LeWitt, P. A., Nyenhuis, D., Olanow, C. W., Rascol, O., Schrag, A., Teresi, J. A., Van Hilten, J. J., \& LaPelle, N. (2007). Movement Disorder Society-sponsored revision of the Unified Parkinson's Disease Rating Scale (MDS-UPDRS): Process, format, and clinimetric testing plan. Movement Disorders, 22, 41-47.

Jankovic, J. (2008). Parkinson's disease: clinical features and diagnosis. Journal of Neurology, Neurosurgery \& Psychiatry, 79, 368-376.

Keus, S. H. J., Nieuwboer, A., Bloem, B. R., Borm, G. F., \& Munneke, M. (2009). Clinimetric analyses of the Modified Parkinson Activity Scale. Parkinsonism and Related Disorders, 15, 263-269.

Koo, T. K., \& Li, M. Y. (2016). A Guideline of Selecting and Reporting Intraclass Correlation Coefficients for Reliability Research. Journal of Chiropractic Medicine, 15, 155-163.

Lakke, J. P. W. F. (1985). Axial apraxia in Parkinson's disease. Journal of the Neurological Sciences, 69, 37-46.

Lau, B., Meier, N., Serra, G., Czernecki, V., Schuepbach, M., Navarro, S., Cornu, P., Grabli, D., Agid, Y., Vidailhet, M., Karachi, C., \& Welter, M. L. (2019). Axial symptoms predict mortality in patients with Parkinson disease and subthalamic stimulation. Neurology, 92, e2559-e2570.

McHugh, M. L. (2012). Interrater reliability: the kappa statistic. Biochemia Medica, 22, 276-282.

Mirelman, A., Hillel, I., Rochester, L., Del Din, S., Bloem, B. R., Avanzino, L., Nieuwboer, A., Maidan, I., Herman, T., Thaler, A., Gurevich, T., Kestenbaum, M., Orr-Urtreger, A., Brys, M., Cedarbaum, J. M., Giladi, N., \& Hausdorff, J. M. (2020). Tossing and Turning in Bed: Nocturnal Movements in Parkinson's Disease. Movement Disorders, 35, 959-968.

Mount, J., Cianci, H., Weiman, R., Da Costa, J., Tabibian, H., \& Prochaska, J. (2009). How People with Parkinson's disease get out of bed. Physical \& Occupational Therapy in Geriatrics, 27, 333-359. 
Mount, J., Kresge, L., Klaus, G., Mann, L., \& Palomba, C. (2006). Movement Patterns Used by the Elderly When Getting Out of Bed. Physical \& Occupational Therapy in Geriatrics, 24, 27-43.

Nieuwboer, A., Weerdt, W. D., Dom, R., \& Lesaffre, E. (1998). A frequency and correlation analysis of motor deficits in Parkinson patients. Disability and Rehabilitation, 20, 142-150.

Pfeiffer, R. F., \& Wszolek, Z. K. (2012). Parkinson's disease (second edition), (940). Florida, USA: CRC Press.

Postuma, R. B., Berg, D., Stern, M., Poewe, W., Olanow, C. W., Oertel, W., Obeso, J., Marek, K., Litvan, I., Lang, A. E., Halliday, G., Goetz, C. G., Gasser, T., Dubois, B., Chan, P., Bloem, B. R., Adler, C. H., \& Deuschl, G. (2015). MDS clinical diagnostic criteria for Parkinson's disease. Movement Disorders, 30, 1591-1601.

Robichaud, J., Pfann, K., Comella, C., Brandabur, M., \& Corcos, D. (2004). Greater impairment of extension movements as compared to flexion movements in Parkinson's disease. Experimental Brain Research, 156, 240-254.

Russell Merritt, Mark H. DeLegge, Beverly Holcombe, Charles Mueller, Juan Ochoa, Karen Ringwald Smith, \& W. Frederick Schwenk II. (2005). American society for parenteral and enteral nutrition. In The A.S.P.E.N. Nutrition support practice manual 2nd edition, 18-19.

Sringean, J., Taechalertpaisarn, P., Thanawattano, C., \& Bhidayasiri, R. (2016). How well do Parkinson's disease patients turn in bed? Quantitative analysis of nocturnal hypokinesia using multisite wearable inertial sensors. Parkinsonism and Related Disorders, 23, 10-16.
Stack, E. L., \& Ashburn, A. M. (2006). Impaired bed mobility and disordered sleep in Parkinson's disease. Movement Disorders, $21,1340-1342$.

Taniguchi, S., Nakata, Y., Inoue, M., \& Marumoto, K. (2021). Validation and Reliability of the Japanese Version of the Modified Parkinson Activity Scale (M-PAS). Progress in Rehabilitation Medicine, 6.

Thorborg, K., Bandholm, T., \& Holmich, P. (2013). Hip- and kneestrength assessments using a hand-held dynamometer with external belt-fixation are inter-tester reliable. Knee Surgery, Sports Traumatology, Arthroscopy, 21, 550-555.

Viwattanakulvanid, P., Kaewwilai, L., Jitkritsadakul, O., Brenden, N., Setthawatcharawanich, S., Boonrod, N., Mekawichai, P., \& Bhidayasiri, R. (2014). The impact of the nocturnal disabilities of Parkinson's disease on caregivers' burden: implications for interventions. Journal of Neural Transmission, 121, 15-24.

Wierzbicka, M. M., Wiegner, A. W., Logigian, E. L., \& Young, R. R. (1991). Abnormal most-rapid isometric contractions in patients with Parkinson's disease. Journal of Neurology, Neurosurgery \& Psychiatry, 54, 210.

Wimmers, R. H., \& YPT, K. (1998). Problematic activities of daily living in patients 12 with Parkinson's disease. Ned Tijdschr Fysiother, 54-56.

Yeo, L., Singh, R., Gundeti, M., Barua, J., \& Masood, J. (2012). Urinary tract dysfunction in Parkinson's disease: a review. International Urology and Nephrology, 44, 415-424. 